\title{
The Benefits of Physical Activity and Positive Mental Health for Reducing the Burden of COVID-19: Validation from a Cross-sectional and Longitudinal Investigation in China and Germany
}

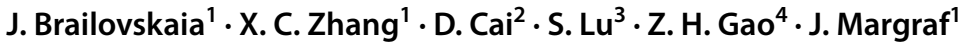

Accepted: 14 September 2021 / Published online: 24 September 2021

(C) The Author(s), under exclusive licence to Springer Science+Business Media, LLC, part of Springer Nature 2021

\begin{abstract}
Many people experience high burden by the outbreak of the coronavirus disease (COVID19) and its consequences for health and everyday life. The present cross-national study investigated potential factors that can reduce the burden by COVID-19 in China and Germany. Cross-sectional and longitudinal (China: $N=474$, baseline, BL: 2015, follow-up, FU: 2020; Germany: $N=359$, BL: 2019, FU: 2020) data on physical activity (e.g., jogging) (BL/FU), positive mental health (PMH) (BL/FU), and burden by COVID-19 (FU) were collected via online surveys. In both countries, physical activity was positively associated with $\mathrm{PMH}$, and both variables were negatively related to burden by COVID-19. Furthermore, PMH mediated the link between physical activity and burden. The mediation model was significant when physical activity and PMH were assessed at the BL, while burden was measured at the FU; and it was also significant when all variables were assessed at the FU. The present findings reveal that physical activity in combination with PMH can reduce the experience of burden by COVID-19. Conscious fostering of physical activity and PMH is supported as an effective strategy to reduce the negative impact of the pandemic outbreak on mental and physical health. Additional benefits such as increased adherence to governmental measures around COVID-19 are discussed.
\end{abstract}

Keyword Physical activity; Positive mental health; COVID-19; Burden; China; Germany

The outbreak of the coronavirus disease 2019 (COVID-19) caused a significant change of everyday life (Fong et al., 2020). By August 31, 2021, there were more than 217,707,000

J. Brailovskaia

Julia.Brailovskaia@rub.de

1 Mental Health Research and Treatment Center, Department of Clinical Psychology and Psychotherapy, Ruhr-Universität Bochum, Massenbergstr. 9-13, 44787 Bochum, Germany

2 Department of Psychology, Shanghai Normal University, Shanghai, China

3 Department of Psychology, Capital Normal University, Beijing, China

4 School of Psychology and Mental Health, North China University of Science and Technology, Qinhuangdao, China 
confirmed COVID-19 cases and more than 4,519,000 deaths caused by COVID-19 worldwide (Beltekian et al., 2021). To fight the pandemic spread, many governments introduced extraordinary measures (World Health Organization, 2020a). Their extent and duration vary across countries but mainly include bans on public gathering and travel, closure of public institutions and non-essential businesses, increased home office, and virtual schooling. The wearing of face masks, the maintaining of distance from other people, and hand disinfection in public places are mandatory behavioral measures in many countries. In some regions, total or overnight curfews belong to the measures (Galea et al., 2020; Sohrabi et al., 2020).

Some people perceive the extraordinary COVID-19 situation and the uncertainty about its duration as a heavy burden (Tang et al., 2021). They experience stress, frustration, anger, and hopelessness (Pfefferbaum \& North, 2020; Xiong et al., 2020). As a consequence, those persons tend to maladaptive coping strategies such as rumination, problematic substance use, and aggressive behavior, and they are at risk to develop insomnia, depression, and anxiety symptoms (Ornell et al., 2020; Taylor et al., 2020). Other individuals perceive the present situation as less burdensome and experience fewer negative emotions (Allington et al., 2020). They search for adaptive coping strategies and maintain a daily routine as far as possible (Brailovskaia \& Margraf, 2020).

The way people perceive and deal with the COVID-19 situation substantially impacts their life at work and in leisure, as well as their mental and physical health. Furthermore, enhanced levels of stress and anxiety can reduce the adherence to actively participate in the governmental COVID-19 prevention measures, while positive emotions increase the cooperation of the population (Margraf et al., 2020). Therefore, it is of great importance to investigate factors and mechanisms that can predict the level of burden caused by the COVID-19 crisis. This knowledge is urgent to identify individuals at risk for high burden and to reduce it. Longitudinally, this can protect mental and physical health and foster adherence to COVID-19 prevention measures (Allington et al., 2020; Salari et al., 2020). While available research often focused on the risk factors such as depression and anxiety symptoms that can enhance the experience of burden (Bäuerle et al., 2020; Ni et al., 2020), only less is currently known about protective factors in the COVID-19 situation. Therefore, the present study aimed to investigate positive variables that have the potential to reduce the COVID-19 burden. The findings could contribute to the development of programs that protect mental and physical health from negative consequences of the current COVID-19 situation.

Physical activity could serve as a protective factor that reduces COVID-19 burden. Notably, following the recommendations of the World Health Organization, (2020b), moderate physical activity (e.g., jogging, cycling) for at least $150 \mathrm{~min}$ throughout a week can significantly contribute to mental and physical health. It increases individual's resilience to adequately cope with stressful experiences and reduces depression, anxiety, and addictive symptoms (Brailovskaia et al., 2018b; Klaperski et al., 2013; Rebar et al., 2015). Steady physical activity improves sleep quality and enhances life satisfaction and academic performance (Eime et al., 2013; Wunsch et al., 2017). Moreover, physical activity reduces suicide-related outcomes (i.e., ideation, behavior) (Brailovskaia et al., 2020; Vancampfort et al., 2018). Therefore, physical activity is often a part of therapeutic treatment of various mental disorders (Firth et al., 2015; Kvam et al., 2016; Schuch et al., 2016).

A further protective factor in the COVID-19 situation might be positive mental health (PMH). PMH comprises facets of emotional, social, and psychological well-being (Lukat et al., 2016). It can reduce the negative impact of daily stress and traumatic experiences (Cai et al., 2017; Truskauskaite-Kuneviciene et al., 2020). Individuals with enhanced levels 
of PMH are less vulnerable to the development of depression and anxiety symptoms and to the negative impact of problematic social media use (Brailovskaia et al., 2019; Teismann et al., 2018; Velten et al., 2018).

Notably, the bulk of resources around governmental responses to the COVID-19 outbreak have gone to expensive measures that are effective but require time for their implementation such as the work on population vaccines, expanding primary healthcare and widespread income support (Margraf et al., 2021; Sunjaya \& Jenkins, 2020). But only little attention has been spent to potential basic protective measures such as physical activity and PMH. Against the available findings (e.g., Brailovskaia et al., 2020; Hu et al., 2020), both could reduce the experienced burden by the COVID-19 situation and support healthy outcomes, as well as the adherence to introduced policies such as quarantine. Therefore, the investigation of the relationship between physical activity, $\mathrm{PMH}$, and burden seems to be both reasonable and urgent.

Based on the presented background, it was expected that physical activity is negatively associated with burden by COVID-19 (Hypothesis 1a); its relationship with PMH was assumed to be positive (Hypothesis 1b); PMH was expected to be negatively related to burden by COVID-19 (Hypothesis 1c). Notably, in a recent longitudinal study, PMH enhanced the protective effect of physical activity on suicide-related outcomes as a mediator over the course of three years (Brailovskaia et al., 2020). Thus, it can be hypothesized that PMH might also interact with physical activity considering the burden by COVID-19. Specifically, PMH could mediate the relationship between physical activity and burden, and thus it could enhance the positive effect of physical activity (Hypothesis 2).

COVID-19 is a global problem that has generated a large interest in international comparisons of governmental responses (Margraf et al., 2020). The present study aimed to investigate the model of how physical activity and PMH can support individuals by reducing the burden in widely different national contexts. It should be tested whether these are universal important protective factors in the COVID-19 crisis. Thus, to gain a broader cross-national view and to go beyond cross-sectional conclusions, it was (1) investigated whether similar result patterns occur in samples from two countries that have a different course of the COVID-19 spread, China and Germany (Beltekian et al., 2021), and (2) data on physical activity and $\mathrm{PMH}$ that were assessed before the pandemic outbreak in the frame of the "Bochum Optimism and Mental Health (BOOM)" program (Margraf \& Schneider, 2017) were combined with data collected after the COVID-19 outbreak to implement a cross-sectional and a longitudinal investigation.

The worldwide first COVID-19 case was reported in China in December 2019. During the year 2020 and the beginning of 2021, the Chinese government imposed quarantine in different regions of the country. Its extent and timeline depended on the number of detected cases in the specific regions. It included measures such as total curfew, closing of schools and universities, temperature checks, wearing of face masks in public places, and keeping of social distance. After a remarkable increase of COVID-19 infections in February 2020, daily case numbers remained relatively low and stable during 2020. On August 31, 2021, a total of 94,898 COVID-19 cases was registered in China (National Health Commission of the People's Republic of China, 2021).

In Germany, the first national quarantine was declared in March 2020. Its timeline and extent differed between the federal states: In some states, curfews were imposed, and other states only encouraged a "stay-at-home". Nationwide, bans on public gatherings and closure of public institutions and non-essential business were introduced. Wearing of face masks in public transport, shops, and buildings with public traffic and keeping of distance remained mandatory after the easing of the quarantine in the end of April 2020. 
During the summer months, daily case numbers remained on a relative low level. However, they remarkably increased during autumn and winter. A second national quarantine was declared in December 2020. Since spring 2021, some of the introduced measures are eased step by step (Bundesministerium für Gesundheit, 2021). On August 31, 2021, a total of 3,965,681 COVID-19 cases was registered in Germany (Beltekian et al., 2021).

\section{Methods}

\section{Procedure and Participants}

Chinese Sample The Chinese sample is comprised of 474 participants $(68.6 \%(n=325)$ women; first measurement time point (baseline, BL): age: $M(S D)=21.66(0.86)$, range: 19-26; occupation: BL: all students; second measurement time point (follow-up, FU): $14.3 \%(n=68)$ students, $84.6 \%(n=401)$ employees, $1.1 \%(n=5)$ unemployed; marital status: BL: $80.4 \%(n=381)$ single, $19.6 \%(n=93)$ with romantic partner; FU: $40.9 \%$ $(n=194)$ single, $35.7 \%(n=169)$ with romantic partner, $23.4 \%(n=111)$ married). At FU, $3.8 \%(n=18)$ reported to belong to the COVID-19 risk group (i.e., age-related, pre-existing condition, weakened immune system), and one person was currently tested positive for COVID-19. In May 2020, an invitation including a link leading to the online survey was emailed to 2,723 persons. They all are current or former students at large universities in China (i.e., Shanghai Normal University, Capital Normal University, North China University of Science and Technology) who had participated in an online survey in May 2015 in the frame of the "BOOM" program. The survey in March 2015 served as BL and included data on physical activity and PMH; the survey in May 2020 that was completed by 474 persons and assessed physical activity, PMH, and the experience of burden by COVID-19 served as FU.

German Sample The German sample is comprised of 359 participants $(76.9 \%(n=276)$ women; BL: age: $M(S D)=25.73$ (7.28), range: $18-70$; occupation: BL: $85 \%(n=305)$ students, $14.8 \%(n=53)$ employees, one unemployed person; FU: $73.5 \%(n=264)$ students, 25.3\% $(n=91)$ employees, $1.1 \%(n=4)$ unemployed; marital status: BL: $46.2 \%(n=166)$ single, $42.1 \%(n=151)$ with romantic partner, $11.7 \%(n=42)$ married; FU: $42.3 \%(n=152)$ single, $42.1 \%(n=151)$ with romantic partner, $15.6 \%(n=56)$ married). At FU, $12.8 \%$ $(n=46)$ reported to belong to the COVID-19 risk group, and three persons were currently tested positive for COVID-19. In November 2020, an invitation including a link leading to the online survey was emailed to 391 persons who had participated in an online survey in November 2019 in the frame of the "BOOM" program. They all are current or former students at a large university in the Ruhr region of Germany. The survey in November 2019 served as BL and included data on physical activity and PMH; the survey in November 2020 that was completed by 359 persons and assessed physical activity, $\mathrm{PMH}$, and the level of COVID-19 burden served as FU.

In both countries, there were no specific requirements for participation that was voluntary and not compensated. The present study received an approval by the responsible Ethics Committees in Germany and in China. The study was conducted in accordance with the principles outlined in the Declaration of Helsinki. All participants were properly instructed 
and gave informed consent to participate via an online form. Independent $t$-tests revealed no significant differences considering the variables of interest between responders (participation at BL and FU) and non-responders (participation only at BL). The surveys were provided in the national language of the samples. There were no missing data. Power analyses $\left(G^{*}\right.$ Power program, version 3.1) revealed that both sample sizes are sufficient for valid results (power $>0.80, \alpha=0.05$, effect size: $f^{2}=0.15$; cf., Mayr, Erdfelder, Buchner, \& Faul, 2007).

\section{Measures}

Frequency of Physical Activity The frequency of physical activity was measured using the item "How frequently did you engage in physical activity (e.g., jogging, cycling) in the last 12 months?" that is rated on a 5-point Likert-type scale $(0=$ never, $4=$ four times $a$ week or more). Following previous research, this item is a reliable and valid instrument to measure physical activity (Brailovskaia et al., 2018b; Milton et al., 2011).

Positive Mental Health (PMH) The unidimensional Positive Mental Health Scale (PMHScale; Lukat et al., 2016) assessed PMH with nine items that are rated on a 4-point Likerttype scale (e.g., "I enjoy my life"; $0=$ do not agree, $3=$ agree). Previous research reported a scale reliability of Cronbach's $\alpha=0.930$ for the PMH-Scale (Lukat et al., 2016). In the present study, the scale reliability was as follows: China: $\alpha_{\mathrm{BL}}=0.920, \alpha_{\mathrm{FU}}=0.920$; Germany: $\alpha_{\mathrm{BL}}=0.910, \alpha_{\mathrm{FU}}=0.930$. Higher sum scores indicate higher levels of $\mathrm{PMH}$.

Burden Caused by COVID-19 Following Brailovskaia and Margraf (2020), the experience of burden caused by COVID-19 was measured with six items (e.g., "I feel restricted in my everyday life", "I am burdened by the current social situation") that are rated on a 7-point Likert-type scale $(1=I$ do not agree, $7=I$ totally agree $)$. Earlier research reported a scale reliability of $\alpha=0.750$ for this instrument (Brailovskaia \& Margraf, 2020). In the present study, the scale reliability was as follows: China: $\alpha_{\mathrm{FU}}=0.632$, Germany: $\left.\alpha_{\mathrm{FU}}=0.767\right)$. The higher the sum score, the higher the level of burden.

\section{Statistical Analyses}

Statistical analyses were conducted using SPSS 26 and the macro Process version 3.5 (www.processmacro.org/index.html; Hayes, 2021). After descriptive analyses, the relationship between physical activity, PMH, and burden by COVID-19 was assessed by zeroorder bivariate correlation analyses. Next, two mediation models were calculated (process: model 4). In the first model, physical activity (BL) was included as predictor in the model, $\mathrm{PMH}(\mathrm{BL})$ as mediator, and burden (FU) as outcome; considering the relatively young and mostly female composition of both samples, age (BL) and gender (BL) served as covariates. In the second model, all variables were assessed at FU. In both models, path $a$ denoted the association between physical activity and $\mathrm{PMH}$; the relationship between $\mathrm{PMH}$ and burden was denoted by path $b$. The indirect effect $(a b)$ was represented by the combined effect of path $a$ and path $b$. The indirect effect is considered significant when the confidence interval does not include zero. The basic relationship between physical activity and burden was denoted by path $c$ (the total effect), and path $c^{\prime}$ (the direct effect) denoted their association after the inclusion of PMH in the model. The mediation effect was assessed by 
the bootstrapping procedure $(10,000$ samples $)$ that provides percentile bootstrapped confidence intervals (CI 95\%).

All calculations were conducted in both country-specific samples.

\section{Results}

Table 1 shows descriptive statistics and correlations of the investigated variables. In both countries, a similar result pattern occurred. Physical activity (BL) was significantly positively correlated with $\mathrm{PMH}(\mathrm{BL})$; physical activity (FU) was significantly positively correlated with PMH (FU); physical activity (BL/FU) and PMH (BL/FU) were both significantly negatively correlated with burden by COVID-19 (FU). Furthermore, physical activity (BL) and physical activity (FU) were significantly positively correlated, as well as $\mathrm{PMH}(\mathrm{BL})$ and PMH (FU).

The mediation analyses revealed similar findings in both country-specific samples. The findings were also similar for both models (i.e., model 1 included physical activity and $\mathrm{PMH}$ at BL, and model 2 included all variables at FU).

As shown in Fig. 1a, in the first mediation model in China, PMH (BL, mediator) significantly mediated the negative association between physical activity (BL, predictor) and burden by COVID-19 (FU, outcome). The basic relationship between physical activity (BL) and burden (FU) was significant (total effect, $c: p=0.011$ ). The relationship between physical activity (BL) and PMH (BL) $(a: p<0.001)$ and the relationship between PMH (BL) and burden $(\mathrm{FU})(b: p<0.001)$ were also significant. After the inclusion of $\mathrm{PMH}(\mathrm{BL})$ in the model, the association between physical activity (BL) and burden (FU) was not significant (direct effect, $c^{\prime}: p=0.250$ ). The indirect effect was significant $(a b), b=-0.331$, $\mathrm{SE}=0.091,95 \% \mathrm{CI}[-0.523,-0.170]$.

Figure $1 \mathrm{~b}$ shows the findings of the second mediation model in China. PMH (FU) significantly mediated the negative link between physical activity (FU) and burden by COVID-19 (FU). The basic association between physical activity (FU) and burden (FU)

Table 1 Descriptive statistics and correlations of the investigated variables (baseline and follow-up)

\begin{tabular}{|c|c|c|c|c|c|c|}
\hline & $M(S D)$ & Min-Max & (2) & (3) & (4) & (5) \\
\hline \multicolumn{7}{|l|}{ China $(N=474)$} \\
\hline (1) Physical activity (BL) & $3.23(1.13)$ & $1-5$ & $.245^{* *}$ & $.360 * *$ & $.207 * *$ & $-.091 *$ \\
\hline (2) Positive mental health (BL) & $20.79(4.69)$ & $4-27$ & & $.193 * *$ & $.501 * *$ & $-.251^{* *}$ \\
\hline (3) Physical activity (FU) & $3.03(1.12)$ & $1-5$ & & & $.284 * *$ & $-.095^{*}$ \\
\hline (4) Positive mental health (FU) & $19.67(4.98)$ & $0-27$ & & & & $-.429 * *$ \\
\hline (5) Burden by COVID-19 (FU) & $17.76(5.74)$ & $6-42$ & & & & \\
\hline \multicolumn{7}{|l|}{ Germany $(N=359)$} \\
\hline (1) Physical activity (BL) & $2.95(1.14)$ & $1-5$ & $.242 * *$ & $.297 * *$ & .090 & $-.107 *$ \\
\hline (2) Positive mental health (BL) & $17.77(5.72)$ & $0-27$ & & $.240 * *$ & $.454 * *$ & $-.168 * *$ \\
\hline (3) Physical activity (FU) & $3.12(1.23)$ & $1-5$ & & & $.270^{* *}$ & $-.113^{*}$ \\
\hline (4) Positive mental health (FU) & $18.07(6.08)$ & $1-27$ & & & & $-.422 * *$ \\
\hline (5) Burden by COVID-19 (FU) & $23.44(6.75)$ & $7-40$ & & & & \\
\hline
\end{tabular}

Note. $M$ mean, SD standard deviation, Min minimum, Max maximum, $B L$ baseline; $F U$ follow-up; $* * p<.01 ; * p<.05$ 

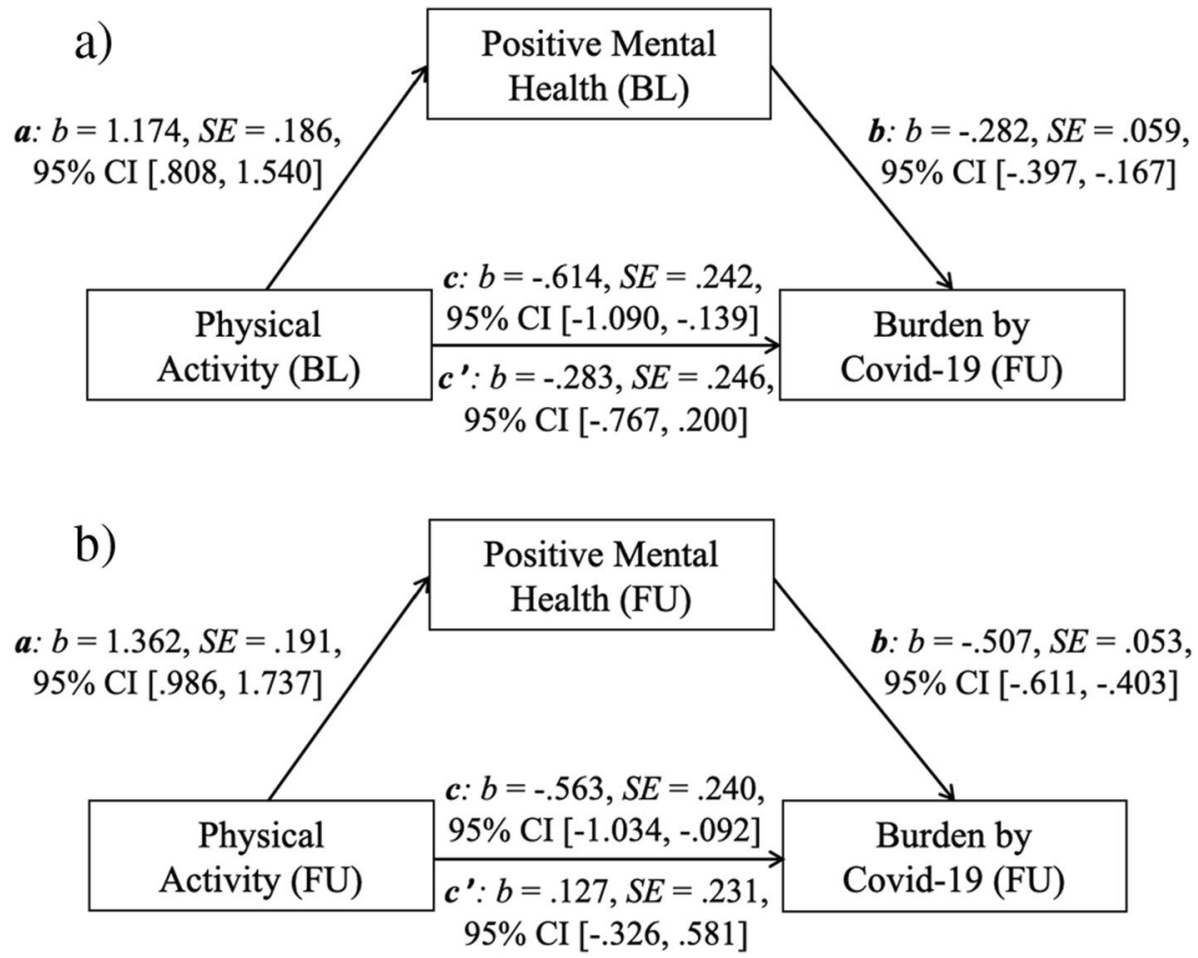

Fig. 1 Mediation model Chinese sample including physical activity (predictor), positive mental health (mediator), and burden caused by COVID-19 (outcome): a physical activity and positive mental health assessed at baseline, burden assessed at follow-up; $\mathbf{b}$ all variables assessed at follow-up. Notes. $\mathrm{c}=$ path of predictor to outcome, without inclusion of mediator (total effect); $a=$ path of predictor to mediator; $b=$ path of mediator to outcome; c'= path of predictor to outcome including mediator (direct effect); BL, baseline; FU, follow-up

was significant (total effect, $c: p=0.019)$. The association between physical activity (FU) and PMH (FU) $(a: p<0.001)$ and the relationship between PMH (FU) and burden (FU) $(b$ : $p<0.001$ ) were also significant. After the inclusion of PMH (FU) in the model, the association between physical activity (FU) and burden (FU) was not significant (direct effect, $\left.c^{\prime}: p=0.581\right)$. The indirect effect was significant $(a b), b=-0.691, \mathrm{SE}=0.130,95 \% \mathrm{CI}$ $[-0.957,-0.450]$.

The same result pattern occurred in Germany as in China (see Fig. 2). In the first model in Germany (see Fig. 2a), PMH (BL) significantly mediated the negative relationship between physical activity (BL) and burden by COVID-19 (FU). The basic relationship between physical activity (BL) and burden (FU) was significant (total effect, $c: p=0.012$ ). The association between physical activity (BL) and PMH (BL) $(a: p<0.001)$ and the relationship between PMH (BL) and burden (FU) $(b: p=0.004)$ were also significant. After the inclusion of PMH (BL) in the model, the link between physical activity (BL) and burden (FU) was not significant (direct effect, $c^{\prime}: p=0.071$ ). The indirect effect was significant $(a b)(b=-0.218, \mathrm{SE}=0.093,95 \% \mathrm{CI}[-0.425,-0.060])$.

The results of the second mediation model in Germany are shown in Fig. 2b. PMH (FU) significantly mediated the negative link between physical activity (FU) and burden 

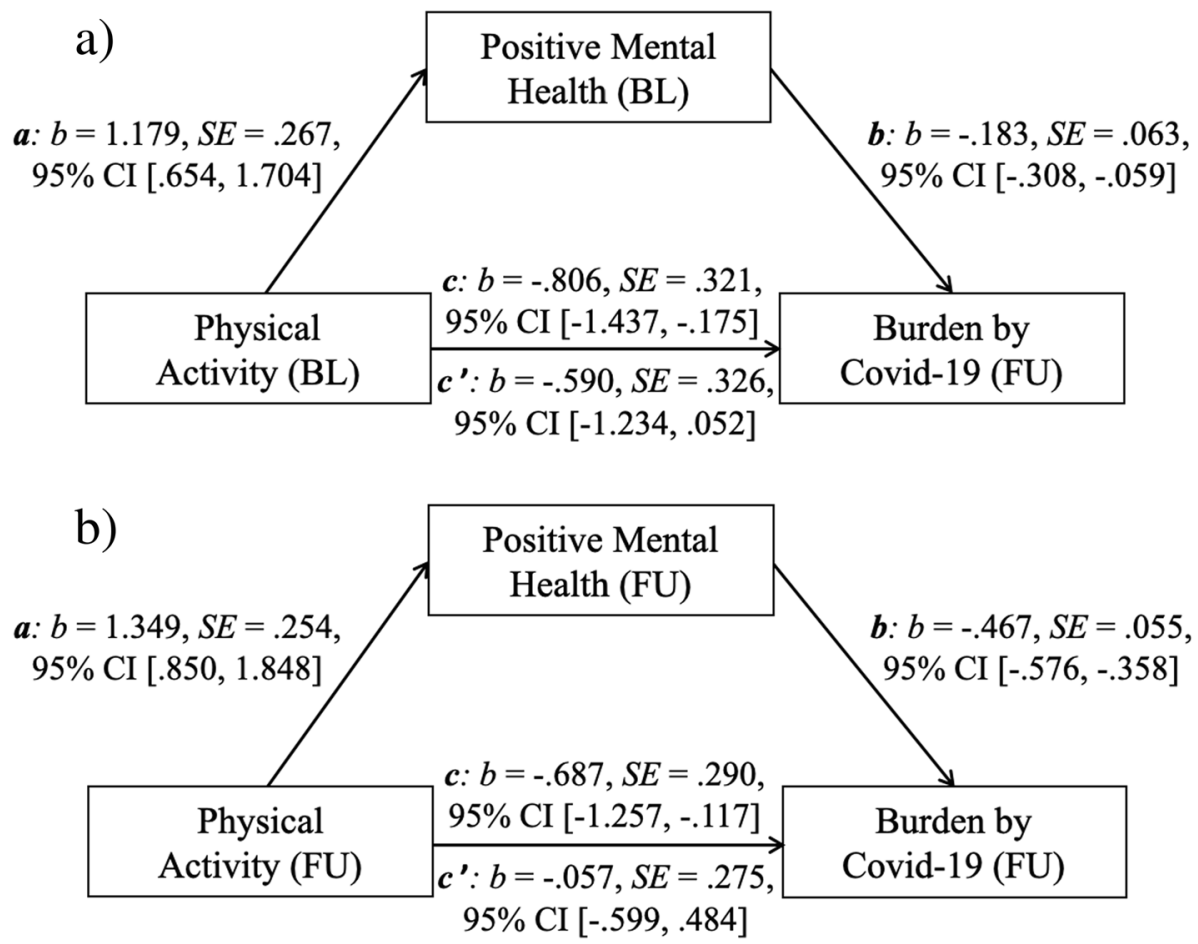

Fig. 2 Mediation model German sample including physical activity (predictor), positive mental health (mediator), and burden caused by COVID-19 (outcome): a physical activity and positive mental health assessed at baseline, burden assessed at follow-up; b all variables assessed at follow-up. Notes. $\mathrm{c}=$ path of predictor to outcome, without inclusion of mediator (total effect); $a=$ path of predictor to mediator; $b=$ path of mediator to outcome; c' = path of predictor to outcome including mediator (direct effect); BL, baseline; FU, follow-up

by COVID-19 (FU). The basic association between physical activity (FU) and burden (FU) was significant (total effect, $c: p=0.018$ ). The association between physical activity (FU) and PMH (FU) $(a: p<0.001)$ and the relationship between PMH (FU) and burden (FU) $(b$ : $p<0.001$ ) were also significant. After the inclusion of PMH (FU) in the model, the association between physical activity (FU) and burden (FU) was not significant (direct effect, $\left.c^{\prime}: p=0.835\right)$. The indirect effect was significant $(a b)(b=-0.630, \mathrm{SE}=0.158,95 \% \mathrm{CI}$ $[-0.964,-0.350]$.

\section{Discussion}

The further duration and course of the COVID-19 situation are unclear. Therefore, it is of great importance to identify factors that can reduce its negative impact. The present cross-national study provides cross-sectional and longitudinal findings from China and Germany on two protective factors that could reduce burden experienced by COVID-19, and it explains how they might interact. Despite the different national COVID-19 contexts, similar result patterns were found in both countries. 
As expected, physical activity assessed one (in Germany) and five (in China) years before the pandemic outbreak (longitudinal data), as well as physical activity assessed in both countries after the pandemic outbreak (cross-sectional data), was negatively associated with burden caused by COVID-19 (confirmation of Hypothesis 1a). Regular physical activity (e.g., jogging, cycling, yoga) not only fosters physical health, but also increases the individual level of self-efficacy and confers resilience to deal with stressful and uncertain situations (Eime et al., 2013). In line with this knowledge, the current findings show that individuals who regularly engaged/engage in physical activity in the past and in the present are at less risk to experience high burden by COVID-19. Furthermore, physical activity was positively associated with PMH (confirmation of Hypothesis 1b). This corresponds to earlier research that described that people who engage in physical activity often experience flow — a state of joy, happiness, and mastery (Csikszentmihalyi, 1990) - that fosters positive mental health (Drane \& Barber, 2016; Richards et al., 2015). Furthermore, PMH can be increased by the achieving of selfdetermined physical aims such as the enhancement of own cycling speed and endurance (Brailovskaia et al., 2020).

PMH was negatively associated with COVID-19 burden (confirmation of Hypothesis 1c). Previous studies described the positive impact of PMH in different life areas. For example, it contributed to the longitudinal treatment success of individuals with anxiety disorders (Teismann et al., 2018) and reduced the risk of suicide ideation and behavior in individuals who previously experienced cyberbullying (Brailovskaia et al., 2018a). The current findings reveal that PMH assessed up to five years before the pandemic outbreak as well as PMH measured in the present can predict the level of burden by COVID-19. Moreover, PMH mediated the relationship between physical activity and COVID-19 burden (confirmation of Hypothesis 2). Thus, it can be hypothesized that the protective effect of physical activity against COVID-19 burden depends on heightened PMH: If physical activity translates to $\mathrm{PMH}$, then the experience of high COVID-19 burden becomes less likely. This assumption corresponds to earlier research that showed the combination of physical activity and PMH to be a protective factor against suicide-related outcomes (Brailovskaia et al., 2020). Notably, in the current study, the hypothesis about the mediation role of PMH was confirmed in the longitudinal mediation model that included physical activity and PMH five years (Chinese sample) and one year (German sample) before the pandemic outbreak and in the crosssectional model that included all data at the same measurement time point. Furthermore, there was a positive relationship between physical activity at the BL and at the FU, as well as between $\mathrm{PMH}$ at the $\mathrm{BL}$ and at the FU. The present results reveal the stability of the protective effect of physical activity and PMH. Moreover, they emphasize the importance of the permanent maintenance of both variables on a high level to reduce the impact of negative external factors such as the COVID-19 outbreak.

The present findings contribute to and extend available research on physical activity and PMH (Rebar et al., 2015; Truskauskaite-Kuneviciene et al., 2020). Notably, the different course of the COVID-19 spread (Beltekian et al., 2021), along with the confounding impact of different national profiles and governmental measures in China and Germany (Mitze et al., 2020; Tian et al., 2020), cannot be sorted out. Moreover, there is a gap of four years between the BL assessed in China and the BL assessed in Germany which limits their direct comparability. Therefore, it is especially interesting to see that similar result patterns occurred in both national contexts of COVID-19. This finding confirms the importance of physical activity and PMH as protective factors in extraordinary situations (Eime et al., 2013; Truskauskaite-Kuneviciene et al., 2020) and allows the hypothetical assumption of the universality of their positive effect. 
In this context, one can better understand why some people experience high burden by the current situation, while others react in a more adaptive way. High burden by COVID-19 can contribute to maladaptive behavior such as enhanced alcohol consumption, aggression, and less adherence to governmental measures (Barzilay et al., 2020; Margraf et al., 2020). An early identification of and a targeted providing of support for people who are at risk to experience high COVID-19 burden can reduce the negative consequences which in the longer term harm the individual and the society. For example, freely available online programs could be offered and promoted by official governmental communication that foster physical activity and PMH. Such programs could include instructed gymnastic exercises that can be carried out at home without expensive equipment and are adapted to specific focus groups (e.g., considering age, health status). Online sportive programs that allow participants to see each other and to interact with each other during the exercises could at least partly satisfy the need for belonging and increase the perception of social support. Both are of great importance in the current COVID-19 situation that requires social distancing to fight the pandemic spread (Galea et al., 2020), and both were previously shown to foster the level of PMH (Hu et al., 2020). Furthermore, these programs might contribute to a more regulated daily routine during curfews and for people who must stay in quarantine. They could not only support people who currently suffer from high level of burden, but also preventively reduce the risk of later burden experience.

The following limitations of the current study are to be considered. First, in the present study, only the frequency of the physical activity was assessed. Future research is suggested to include further measures to gain a more detailed overview of physical activity (e.g., which activity is performed; how intensively is it daily performed) and its relationship with PMH and burden. This could contribute to conclusions about the best form and intensity of physical activity to reduce COVID-19 burden. Second, the low internal consistency of the burden measure in the Chinese sample might reduce the reliability of the present results. Third, there was a high drop-out rate in the Chinese sample. Of the overall 2,723 persons who participated in the BL survey, only 474 (17.4\%) responded to the FU survey. The high drop-out can at least partly be explained by the long time period between the BL and the FU measurement. The university email addresses were used to contact the participants. Therefore, it could be that after five years, some of them do not longer check or use this email address. Moreover, previous research reported that especially young adults (between 18 and 23 years) tend to drop-out in longitudinal studies (Young et al., 2006). The mean age of the Chinese sample was 21.66 years at the BL. In contrast, in the German sample, the drop-out rate was relatively low $(8.2 \%, n=32)$ after one year. Notably, in both samples, there were no significant differences considering the demographic variables as well as the levels of physical activity and PMH at the BL between persons who participated in the FU survey and these who did not participate. Furthermore, the levels of physical activity, PMH, and burden in the present study did not remarkably differ from the previously reported levels of these variables (Brailovskaia et al., 2021; Lukat et al., 2016; Schönfeld et al., 2017). Nevertheless, it cannot be controlled for further selective factors (e.g., mental health issues, issues of the living environment, family issues) that might influence whether individuals who participated in the BL survey continued their participation in the FU survey.

In conclusion, the present cross-national study reveals that cross-sectionally and longitudinally physical activity and PMH could reduce burden experienced by the current COVID-19 situation in different national contexts. Thus, the enhancement of physical activity and PMH could strengthen the people in the current uncertain situation, it could decrease maladaptive coping strategies, and it could increase adaptive ones. This could 
foster adherence to the governmental COVID-19 prevention measures, and thus this could contribute to the fight with the pandemic spread. Therefore, reducing the burden of COVID-19 through the fostering of protective factors such as physical activity and PMH should belong to preferred strategies. As with most communicable disease, the COVID-19 response worldwide has been dominated by care and infection prevention including widespread quarantine and shutdowns of public life and economy (Su et al., 2021). The present findings show that a basic approach to health promotion can have a longer-term protective advantage that might reduce the burden of outbreaks such as COVID-19 and thus significantly support the success of the other measures.

Funding This work was supported by the Alexander von Humboldt Foundation and by the Volkswagen Foundation. The funding source had no role in study design, in the collection, analysis and interpretation of data, in the writing of the report, and in the decision to submit the article for publication.

Data Availability The dataset and further material analyzed during the current study will be available from the corresponding author on reasonable request.

\section{Declarations}

Ethical Approval The responsible Ethics Committee approved the present study.

Informed Consent All participants were properly instructed and gave online their informed consent to participate. All procedures followed were in accordance with the ethical standards of the responsible committee on human experimentation (institutional and national) and with the Helsinki Declaration of 1975, as revised in 2000 (5). Informed consent was obtained from all patients for being included in the study.

Consent for Publication All participants were properly instructed that data gained in the present study will be used for publication in an anonymous form and gave online their informed consent for publication.

Conflict of Interest The author declare no competing interests.

\section{References}

Allington, D., Duffy, B., Wessely, S., Dhavan, N., \& Rubin, J. (2020). Health-protective behaviour, social media usage and conspiracy belief during the COVID-19 public health emergency. Psychological Medicine, 1-7. https://doi.org/10.1017/S003329172000224X

Barzilay, R., Moore, T. M., Greenberg, D. M., DiDomenico, G. E., Brown, L. A., White, L. K., \& Gur, R. E. (2020). Resilience, COVID-19-related stress, anxiety and depression during the pandemic in a large population enriched for healthcare providers. Translational Psychiatry, 10(1), 1-8. https://doi.org/10. 1038/s41398-020-00982-4

Bäuerle, A., Steinbach, J., Schweda, A., Beckord, J., Hetkamp, M., Weismüller, B., \& Teufel, M. (2020). Mental health burden of the CoViD-19 outbreak in germany: Predictors of mental health impairment. Journal of Primary Care \& Community Health, 11, 1-8. https://doi.org/10.1177/2150132720953682

Beltekian, D., Gavrilov, D., Giattino, C., Hasell, J., Macdonald, B., Mathieu, E., . . Roser, M. (2021). Covid-19-data. Retrieved from https://github.com/owid/covid-19-data/tree/master/public/data

Brailovskaia, J., Cosci, F., Mansueto, G., Miragall, M., Herrero, R., Baños, R. M., \& Margraf, J. (2021). The association between depression symptoms, psychological burden caused by Covid-19 and physical activity: An investigation in Germany, Italy, Russia, and Spain. Psychiatry Research, 295, 113596. https://doi.org/10.1016/j.psychres.2020.113596

Brailovskaia, J., \& Margraf, J. (2020). Predicting adaptive and maladaptive responses to the coronavirus (COVID-19) outbreak: A prospective longitudinal study. International Journal of Clinical and Health Psychology, 20(3), 181-191. https://doi.org/10.1016/j.ijchp.2020.06.002 
Brailovskaia, J., Rohmann, E., Bierhoff, H.-W., Margraf, J., \& Köllner, V. (2019). Relationships between addictive Facebook use, depressiveness, insomnia, and positive mental health in an inpatient sample: A German longitudinal study. Journal of Behavioral Addictions, 8(4), 703-713. https://doi.org/10.1556/ 2006.8.2019.63

Brailovskaia, J., Teismann, T., \& Margraf, J. (2018). Cyberbullying, positive mental health and suicide ideation/behavior. Psychiatry Research, 267, 240-242. https://doi.org/10.1016/j.psychres.2018.05.074

Brailovskaia, J., Teismann, T., \& Margraf, J. (2018). Physical activity mediates the association between daily stress and Facebook Addiction Disorder (FAD) - a longitudinal approach among German students. Computers in Human Behavior, 86, 199-204. https://doi.org/10.1016/j.chb.2018.04.045

Brailovskaia, J., Teismann, T., \& Margraf, J. (2020). Positive mental health mediates the relationship between physical activity and suicide-related outcomes: A three-year follow-up study. Current Psychology. https://doi.org/10.1007/s12144-020-01152-X

Bundesministerium für Gesundheit. (2021). Coronavirus SARS-CoV-2: Chronik der bisherigen Maßnahmen. Retrieved from https://www.bundesgesundheitsministerium.de/coronavirus/chronik-coronavirus. html

Cai, D., Zhu, M., Lin, M., Zhang, X. C., \& Margraf, J. (2017). The bidirectional relationship between positive mental health and social rhythm in college students: A three-year longitudinal study. Frontiers in Psychology, 8, 1-7. https://doi.org/10.3389/fpsyg.2017.01119

Csikszentmihalyi, M. (1990). Flow: The psychology of optimal performance. Cambridge UniversityPress.

Drane, C. F., \& Barber, B. L. (2016). Who gets more out of sport? The role of value and perceived ability in flow and identity-related experiences in adolescent sport. Applied Developmental Science, 20(4), 267-277. https://doi.org/10.1080/10888691.2015.1114889

Eime, R. M., Young, J. A., Harvey, J. T., Charity, M. J., \& Payne, W. R. (2013). A systematic review of the psychological and social benefits of participation in sport for adults: Informing development of a conceptual model of health through sport. International Journal of Behavioral Nutrition and Physical Activity, 10(1), 135-148. https://doi.org/10.1186/1479-5868-10-135

Firth, J., Cotter, J., Elliott, R., French, P., \& Yung, A. R. (2015). A systematic review and meta-analysis of exercise interventions in schizophrenia patients. Psychological Medicine, 45(7), 1343-1361. https:// doi.org/10.1017/S0033291714003110

Fong, M. W., Gao, H., Wong, J. Y., Xiao, J., Shiu, E. Y. C., Ryu, S., \& Cowling, B. J. (2020). Nonpharmaceutical measures for pandemic influenza in nonhealthcare settings-Social distancing measures. Emerging Infectious Diseases, 26(5), 976-984. https://doi.org/10.3201/eid2605.190995

Galea, S., Merchant, R. M., \& Lurie, N. (2020). The mental health consequences of COVID-19 and physical distancing: The need for prevention and early intervention. JAMA Internal Medicine, 180(6), 817-818. https://doi.org/10.1001/jamainternmed.2020.1562

Hayes, A. (2021). Introduction to Mediation, Moderation, and Conditional Process Analysis (2nd ed.). The Guilford Press.

Hu, S., Cai, D., Zhang, X. C., \& Margraf, J. (2020). Relationship between social support and positive mental health: A three-wave longitudinal study on college students. Current Psychology, 1-10. https://doi.org/ 10.1007/s12144-020-01175-4

Klaperski, S., von Dawans, B., Heinrichs, M., \& Fuchs, R. (2013). Does the level of physical exercise affect physiological and psychological responses to psychosocial stress in women? Psychology of Sport and Exercise, 14(2), 266-274. https://doi.org/10.1016/j.psychsport.2012.11.003

Kvam, S., Kleppe, C. L., Nordhus, I. H., \& Hovland, A. (2016). Exercise as a treatment for depression: A meta-analysis. Journal of Affective Disorders, 202, 67-86. https://doi.org/10.1016/j.jad.2016.03.063

Lukat, J., Margraf, J., Lutz, R., van der Veld, W. M., \& Becker, E. S. (2016). Psychometric properties of the positive mental health scale (PMH-scale). BMC Psychology, 4(1), 8. https://doi.org/10.1186/ s40359-016-0111-x

Margraf, J., Brailovskaia, J., \& Schneider, S. (2020). Behavioral measures to fight COVID-19: An 8-country study of perceived usefulness, adherence and their predictors. PLOS ONE, 15(12), e0243523. https:// doi.org/10.1371/journal.pone.0243523

Margraf, J., Brailovskaia, J., \& Schneider, S. (2021). Adherence to behavioral Covid-19 mitigation measures strongly predicts mortality. PLoS ONE, 16(3), e0249392. https://doi.org/10.1371/journal.pone.02493 92

Margraf, J., \& Schneider, S. (2017). Bochum Optimism and Mental Health (BOOM) research program: Background, methods and aims. Manuscript in preparation.

Mayr, S., Erdfelder, E., Buchner, A., \& Faul, F. (2007). A short tutorial of GPower. Tutorials in Quantitative Methods for Psychology, 3(2), 51-59. https://doi.org/10.20982/tqmp.03.2.p051 
Milton, K., Bull, F. C., \& Bauman, A. (2011). Reliability and validity testing of a single-item physical activity measure. British Journal of Sports Medicine, 45(3), 203-208. https://doi.org/10.1136/bjsm. 2009.068395

Mitze, T., Kosfeld, R., Rode, J., \& Wälde, K. (2020). Face masks considerably reduce COVID-19 cases in Germany. Proceedings of the National Academy of Sciences, 117(51), 32293-32301. https://doi. org/10.1073/pnas.2015954117

National Health Commission of the People's Republic of China. (2021). Outbreak notification. Retrieved from http://www.nhc.gov.cn/xcs/yqfkdt/202109/09a9553d105247dd97d2a49a9653d69e.shtml

Ni, M. Y., Yang, L., Leung, C. M. C., Li, N., Yao, X. I., Wang, Y., \& Liao, Q. (2020). Mental health, risk factors, and social media use during the COVID-19 epidemic and cordon sanitaire among the community and health professionals in Wuhan, China: Cross-sectional survey. JMIR Mental Health, 7(5), e19009. https://doi.org/10.2196/19009

Ornell, F., Schuch, J. B., Sordi, A. O., \& Kessler, F. H. P. (2020). "Pandemic fear" and COVID-19: Mental health burden and strategies. Brazilian Journal of Psychiatry, 42(3), 232-235. https://doi.org/ 10.1590/1516-4446-2020-0008

Pfefferbaum, B., \& North, C. S. (2020). Mental health and the Covid-19 pandemic. New England Journal of Medicine, 383, 510-512. https://doi.org/10.1056/NEJMp2008017

Rebar, A. L., Stanton, R., Geard, D., Short, C., Duncan, M. J., \& Vandelanotte, C. (2015). A meta-metaanalysis of the effect of physical activity on depression and anxiety in non-clinical adult populations. Health Psychology Review, 9(3), 366-378. https://doi.org/10.1080/17437199.2015.1022901

Richards, J., Jiang, X., Kelly, P., Chau, J., Bauman, A., \& Ding, D. (2015). Don't worry, be happy: Cross-sectional associations between physical activity and happiness in 15 European countries. BMC Public Health, 15(1), 53. https://doi.org/10.1186/s12889-015-1391-4

Salari, N., Hosseinian-Far, A., Jalali, R., Vaisi-Raygani, A., Rasoulpoor, S., Mohammadi, M., \& Khaledi-Paveh, B. (2020). Prevalence of stress, anxiety, depression among the general population during the COVID-19 pandemic: A systematic review and meta-analysis. Globalization and Health, 16(1), 1-11. https://doi.org/10.1186/s12992-020-00589-w

Schönfeld, P., Brailovskaia, J., \& Margraf, J. (2017). Positive and negative mental health across the lifespan: A cross-cultural comparison. International Journal of Clinical and Health Psychology, 17(3), 197-206. https://doi.org/10.1016/j.ijchp.2017.06.003

Schuch, F. B., Vancampfort, D., Richards, J., Rosenbaum, S., Ward, P. B., \& Stubbs, B. (2016). Exercise as a treatment for depression: A meta-analysis adjusting for publication bias. Journal of Psychiatric Research, 77, 42-51. https://doi.org/10.1016/j.jpsychires.2016.02.023

Sohrabi, C., Alsafi, Z., O’Neill, N., Khan, M., Kerwan, A., Al-Jabir, A., \& Agha, R. (2020). World Health Organization declares global emergency: A review of the 2019 novel coronavirus (COVID19). International Journal of Surgery, 76, 71-76. https://doi.org/10.1016/j.ijsu.2020.02.034

Su, Z., Wen, J., McDonnell, D., Goh, E., Li, X., Šegalo, S., \& Xiang, Y.-T. (2021). Vaccines are not yet a silver bullet: The imperative of continued communication about the importance of COVID-19 safety measures. Brain, Behavior, \& Immunity-Health, 12, 100204. https://doi.org/10.1016/j.bbih. 2021.100204

Sunjaya, A. P., \& Jenkins, C. (2020). Rationale for universal face masks in public against COVID-19. Respirology, 25(7), 678-679. https://doi.org/10.1111/resp.13834

Tang, F., Liang, J., Zhang, H., Kelifa, M. M., He, Q., \& Wang, P. (2021). COVID-19 related depression and anxiety among quarantined respondents. Psychology \& Health, 36(2), 164-178. https://doi.org/ 10.1080/08870446.2020.1782410

Taylor, S., Landry, C. A., Paluszek, M. M., Fergus, T. A., McKay, D., \& Asmundson, G. J. G. (2020). COVID stress syndrome: Concept, structure, and correlates. Depression and Anxiety, 37(8), 706714. https://doi.org/10.1002/da.23071

Teismann, T., Brailovskaia, J., Totzeck, C., Wannemüller, A., \& Margraf, J. (2018). Predictors of remission from panic disorder, agoraphobia and specific phobia in outpatients receiving exposure therapy: The importance of positive mental health. Behaviour Research and Therapy, 108, 40-44. https://doi.org/10.1016/j.brat.2018.06.006

Tian, H., Liu, Y., Li, Y., Wu, C.-H., Chen, B., Kraemer, M. U. G., \& Yang, Q. (2020). An investigation of transmission control measures during the first 50 days of the COVID-19 epidemic in China. Science, 368(6491), 638-642. https://doi.org/10.1126/science.abb6105

Truskauskaite-Kuneviciene, I., Kazlauskas, E., Ostreikaite-Jurevice, R., Brailovskaia, J., \& Margraf, J. (2020). Positive mental health and adjustment following life-stressors among young adults. Current Psychology. https://doi.org/10.1007/s12144-020-00714-3 
Vancampfort, D., Hallgren, M., Firth, J., Rosenbaum, S., Schuch, F. B., Mugisha, J., \& Stubbs, B. (2018). Physical activity and suicidal ideation: A systematic review and meta-analysis. Journal of Affective Disorders, 225, 438-448. https://doi.org/10.1016/j.jad.2017.08.070

Velten, J., Bieda, A., Scholten, S., Wannemüller, A., \& Margraf, J. (2018). Lifestyle choices and mental health: A longitudinal survey with German and Chinese students. BMC Public Health, 18(1), 632. https://doi.org/10.1186/s12889-018-5526-2

World Health Organization. (2020a). Coronavirus disease 2019 (COVID-19): Situation Report, 51. Retrieved from https://apps.who.int/iris/bitstream/handle/10665/331475/nCoVsitrep11Mar2020-eng. pdf

World Health Organization. (2020b). Physical activity. Retrieved from https://www.who.int/news-room/factsheets/detail/physical-activity

Wunsch, K., Kasten, N., \& Fuchs, R. (2017). The effect of physical activity on sleep quality, well-being, and affect in academic stress periods. Nature and Science of Sleep, 9, 117-126. https://doi.org/10.2147/ NSS.S 132078

Xiong, J., Lipsitz, O., Nasri, F., Lui, L. M. W., Gill, H., Phan, L., \& Majeed, A. (2020). Impact of COVID19 pandemic on mental health in the general population: A systematic review. Journal of Affective Disorders, 277(1), 55-64. https://doi.org/10.1016/j.jad.2020.08.001

Young, A. F., Powers, J. R., \& Bell, S. L. (2006). Attrition in longitudinal studies: Who do you lose? Australian and New Zealand Journal of Public Health, 30(4), 353-361. https://doi.org/10.1111/j.1467842X.2006.tb00849.x

Publisher's Note Springer Nature remains neutral with regard to jurisdictional claims in published maps and institutional affiliations. 